\title{
Tratamiento de las verrugas genitales: una actualización
}

\author{
Gerardo González Martínez ${ }^{1}$, José Núñez Troconis. ${ }^{1,2}$ \\ ${ }^{1}$ Departamento de Obstetricia y Ginecología, Hospital “Dr. Manuel Noriega Trigo”, IVSS, Maracaibo, Zulia. ${ }^{2}$ Departamento \\ de Obstetricia y Ginecología, Facultad de Medicina, Universidad del Zulia, Maracaibo, Zulia. Venezuela.
}

\section{RESUMEN}

La infección por el Virus del Papiloma Humano es una afección altamente prevalente a nivel mundial y una de sus consecuencias es la verruga genital externa o condiloma genital. El tratamiento de estas lesiones ha sido tradicionalmente difícil, debido a la alta recurrencia de las lesiones. Se revisan los aspectos más novedosos del tratamiento de las verrugas genitales. Las sinecatequinas y una nueva formulación de Imiquimod al $3,75 \%$ resaltan como las terapéuticas más novedosas, mientras que la terapia fotodinámica carece de evidencia científica adecuada como para recomendar su uso rutinario. Las vacunas profilácticas y terapéuticas parecen poseer un gran potencial pero aún se encuentran en fases iniciales de investigación. Es deseable estudios con un mayor número de pacientes y un seguimiento más prolongado, que permita una comparación directa de la efectividad entre las diferentes técnicas terapéuticas a corto y largo plazo.

\section{PALABRAS CLAVES: Virus del Papiloma Humano, verruga genital, condiloma acuminado, tratamiento}

\section{SUMMARY}

Human Papillomavirus infection is a highly prevalent condition worldwide whose frequent consequence is the external genital wart or genital condyloma. The treatment for this condition has been traditionally difficult due to the recurrence of the lesions. The most innovative aspects of the treatment of genital wart, are reviewed. The sinecatechins and a new formulation of Imiquimod 3.75\% stand out as the most innovative therapeutic, while photodynamic therapy lacks adequate scientific evidence to recommend its routine use. The prophylactic and therapeutic vaccines seem to have great potential but are still in early stages of research. Studies should be conducted with larger patient samples and longer follow-ups to allow comparison of the effectiveness among the different therapeutic techniques in the short and long term.

\section{KEY WORDS: Human papillomavirus, genital wart, condyloma acuminated, treatment}

\section{INTRODUCCIÓN}

La condilomatosis genital o verruga genital externa (VGE) es una infección de transmisión sexual (ITS), altamente contagiosa, causada por el Virus del Papiloma Humano (VPH). El VPH infecta el epitelio basal a través de micro traumas de la piel/ mucosa ano-genital y mucosa oral $(1,2)$. Existe una gran cantidad de diferentes genotipos del VPH, hasta la fecha se han identificado más de 180 diferentes genotipos (3). Los más importantes son aquellos identificados con los números 16 y 18, debido a su reconocido potencial oncogénico, mientras que los genotipos identificados como 6 y 11 y 
que poseen un bajo riesgo oncogénico, son los responsables de las verrugas anogenitales, tanto en mujeres como en hombres. Otros genotipos están relacionados con la aparición de verrugas en otros sitios anatómicos, tales como la planta y palma de pies y manos, dorso de las manos, cara y mucosas.

A pesar de que la VGE es una de las ITS más frecuentes $(4,5)$ su epidemiología no está bien caracterizada. En la población general la incidencia anual de cualquier VGE (incluyendo nuevas y recurrentes) varía desde 160 en España (6) a 289 en el Reino Unido (7) por 100.000 habitantes, con una media de 194,5 por 100.000 habitantes (8).

La distribución regional de nuevos casos de VGE por 100.000 habitantes es la siguiente: 101 a 205 en Norteamérica (9,10), 118 a 170 en Europa (11) y 204 en Asia (11). En relación a la prevalencia, el rango se sitúa entre $0,13 \%$ y $0,16 \%$ en estudios utilizando la población general femenina como denominador $(6,12)$. Los datos epidemiológicos confirman que el pico de incidencia en hombres y mujeres jóvenes corresponde al pico de edad de adquisición de nuevas parejas sexuales (13). Sin embargo, algunos porcentajes de incidencia deben ser interpretados con cautela debido a variaciones en la metodología en cuanto a rangos de edad de las poblaciones (8). Wellings y cols. (13), reportaron una incidencia de 149 por 100.000 habitantes en mujeres con edades comprendidas entre 14 a 65 años de edad comparados con 99 por 100.000 habitantes para la población general femenina (14).

En Venezuela, Ávila y cols. (15), analizaron 90 muestras de lesiones del área genital de 40 hombres y 50 mujeres, sugestivas de infección por el VPH. El análisis histopatológico de las biopsias mostró, que de los 90 pacientes, 72 (80\%) correspondieron a condilomas acuminados, 29 en hombres y 43 en mujeres. El resto de las muestras analizadas $(20 \%)$ presentaron otras patologías, sin embargo, ellos no aportan datos epidemiológicos de Venezuela en este estudio.

Todos los estudios revisados hacen el comentario que se podría estar ante la presencia de un sub-registro, tomando en cuenta que los datos disponibles son limitados debido a que la VGE, a diferencia de otras ITS, no es obligatorio su reporte a las autoridades sanitarias.

El VPH es un virus de ADN de doble cadena que codifica 8 genes (3). L1 y L2 codifican las proteínas estructurales que forman la cápside y E6 y E7 codifican oncoproteínas que actúan interfiriendo la actividad normal de los genes p53 (impidiendo la apoptosis) y pRB (deteniendo el ciclo celular), respectivamente. La expresión combinada de estas proteínas facilita la inestabilidad genómica y cromosómica. El último paso en la carcinogénesis es la integración del ADN vírico en el genoma celular (16).

\section{TIPOS DE VPH}

Mas de 180 tipos de VPH han sido identificados a la fecha, 40 de los cuales infectan el tracto anogenital $(17,3)$. Los tipos son categorizados como de alto o de bajo riesgo, basado en su potencial oncogénico para causar cáncer de cuello uterino. Los tipos de VPH que causan VGE no son los mismos que aquellos que causan cáncer cervical (18).

A nivel mundial, los tipos de VPH denominados 16 y 18 son los responsables de la mayoría de los canceres cervicales y están también asociados con otros canceres ano genitales, incluyendo vulva, vagina, pene y ano, así como también algunos cánceres oro-faríngeos $(19,3)$.

Los VPH tipo 6 y 11 son denominados de bajo riesgo oncogénico, y son estos tipos los que causan el $90 \%$ de las VGE (17). Las pruebas para detección de los tipos de VPH están disponibles, pero solo están indicadas para mujeres quienes son sometidas a tamizaje de cáncer cérvico-uterino (17).

Según la International Council on The Taxonomy of Viruses clasifica los genotipos 6 y 11 dentro del género Alfa-Papillomavirus y están implicados además en la producción de los condilomas acuminados y papilomas del cuello uterino (Figuras 1 y 2) y de la laringe (20).

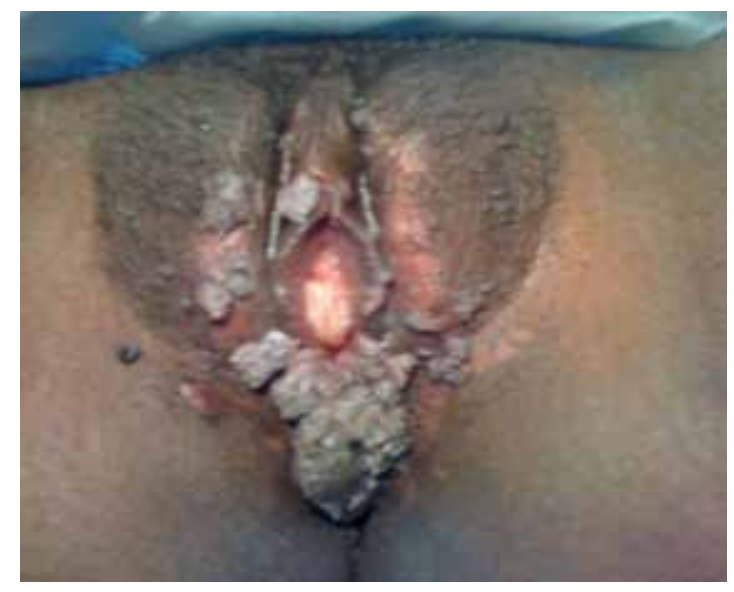

Figura 1. Condilomatosis severa anogenital. Archivo personal. Dr. Núñez Troconis. 


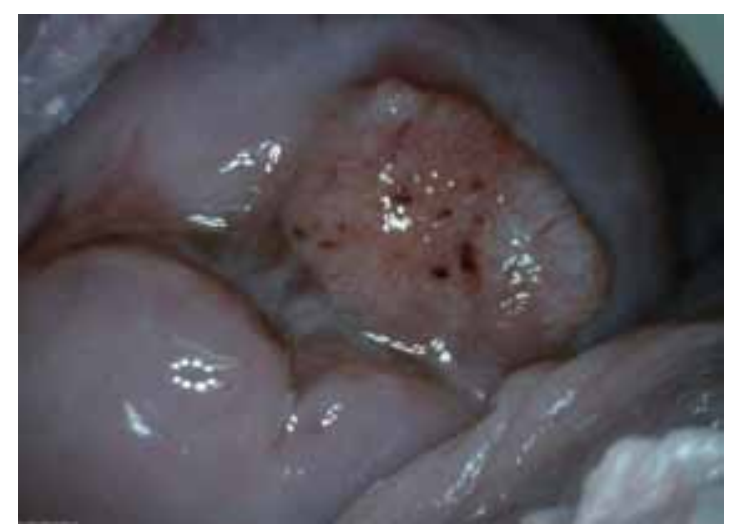

Figura 2. Papiloma de cuello uterino. Archivo personal. Dr. González Martínez.

En la mayoría de los casos, la infección por VPH es asintomática y es el sistema inmune quien se encarga de mantener al VPH indetectable por lo menos durante dos años (21). La infección por VPH es altamente prevalente, más del $50 \%$ de las personas sexualmente activas se infectaran por lo menos una vez en su vida (22). La persistencia de la infección por VPH de alto riesgo (VPH-AR) crea un ambiente adecuado para el desarrollo del cáncer. La co-infección con genotipos de alto y bajo riesgo oncogénico también es posible. Infecciones múltiples incrementa el riesgo de persistencia de la infección y por consiguiente de anomalías citológicas.

\section{CARACTERÍSTICAS CLÍNICAS DE LA VGE}

El diagnóstico diferencial de las VGE incluye a otras condiciones como: condiloma lata, molusco contagioso, liquen nitidus, keratosis seborreica, carcinoma verrucoso, micropapilomatosis labial. La apariencia típica de la VGE es una lesión parecida a la coliflor, pero puede ser plana, papular, keratosica, con capas gruesas o de apariencia frondosa (Figura 1).

La VGE puede desarrollarse en la vulva, perineo o en la piel perianal. Pueden ser asintomáticas o causar sensación de prurito anogenital, ardor y/o dispareunia. En algunos pacientes las VGE se pueden desarrollar en múltiples sitios tales como vagina, cuello uterino, labios y/o paladar.

\section{DIAGNÓSTICO DE LA VGE}

El diagnóstico de la VGE es logrado principalmente por la inspección visual. Una biopsia es necesaria solo en los siguientes casos: paciente inmunocomprometido, diagnóstico incierto, crecimiento repentino de la lesión o lesiones, y lesiones pigmentadas, induradas, ulceradas o sangrantes. La prueba del ácido acético tiene valor predictivo positivo bajo, y las nuevas guías del Centers for Disease Control and Prevention (CDC) no recomiendan este test para el diagnóstico (23).

\section{IMPLICANCIAS DE LA VGE}

El VPH es transmitido a través del contacto genital, usualmente durante el coito genital y/o anal, pero también puede ser transmitido a través del sexo oral. Muchas, sino la mayoría de las personas infectadas desconocen esta condición, y de esa manera, la infección puede ser transmitida a la pareja sexual. Una complicación adicional a esta infección es el largo periodo de incubación desde que ocurre la infección hasta que aparecen los síntomas, lo cual puede estar en el rango de 2 semanas a 8 meses (24).

El impacto psicosocial de la VGE es considerable. Puede incluir rabia, depresión y vergüenza. Muchas pacientes sienten miedo de ser estigmatizadas al ser esta una ITS, y esto frecuentemente tiene un impacto negativo en su relación de pareja y disfrute de la actividad sexual. Puede haber dispareunia y miedo de transmitir la enfermedad a la pareja, así como también miedo de la posibilidad de que la infección por VPH progrese a cáncer (25).

\section{OPCIONES PARA EL TRATAMIENTO DE LA VGE}

La nueva guía de manejo práctico desarrollada por el CDC en 2010, aporta una valiosa información en cuanto a las VGE y su manejo terapéutico, y enfatiza que ninguna terapia es superior a otra para el tratamiento (23). La mejor forma de asegurar el éxito es tener diferentes opciones terapéuticas disponibles con el fin de adaptar cada tratamiento a un paciente en particular basándose en la presentación clínica y en las preferencias personales de cada paciente. Con frecuencia, un tratamiento puede ser usado para obtener un resultado parcial y un segundo tratamiento para completar la curación de las lesiones remanentes.

El objetivo principal del tratamiento de la VGE es la erradicación de las lesiones con el fin de aminorar los síntomas. La paciente puede quejarse de ardor y prurito o sufrir sobre-infección de la lesión, pero la presencia de lesiones anormales solas aporta suficiente evidencia para iniciar el tratamiento. La paciente necesita entender dos puntos antes de que el médico inicie la terapia, especialmente si las lesiones son pocas o pequeñas: a. La observación puede ser adecuada tomando en cuenta que el porcentaje de regresión es relativamente alto y que alcanza hasta un $30 \%$, y aun sin tratamiento las lesiones pueden resolverse espontáneamente (26). b. La remoción de las verrugas no está diseñada para eliminar la infección por VPH. Este 
mensaje es particularmente importante debido al hecho de que las pacientes están aprendiendo más cada día acerca de la infección por VPH, gracias a las herramientas digitales de información principalmente la Internet.

\section{OPCIONES ESPECÍFICAS DE TRATAMIENTO DE LA VGE}

Los tratamientos disponibles para el tratamiento se clasifican por: el mecanismo de acción del fármaco, médico o quirúrgico, y tipo de centro médico donde se aplicara la terapia. EI CDC enfoca sus tratamientos dentro de aquellas terapias que son aplicadas por la paciente y aquellas aplicadas por el médico.

La selección del plan de tratamiento inicial para cualquier individuo depende de diversas variables. Es importante considerar para la elección del tratamiento de las VGE la morfología, el número de lesiones y la localización anatómica. Las verrugas en superficies húmedas o áreas intertriginosas es más probable que respondan al tratamiento local. AqueIlas que son pediculadas son fáciles de extirpar con una simple escisión en la interfase de la verruga y la piel sana. Verrugas múltiples y muy queratinizadas requieren con frecuencia terapia ablativa.

En relación a la paciente el tratamiento dependerá de variables tales como su habilidad para adherirse exitosamente el tratamiento, bien sea aplicándolo en el hogar o regresando a visitas adicionales con el médico tratante, el impacto de los potenciales efectos colaterales, costo en tiempo y dinero, cobertura por las compañías de seguros (algunas compañías aseguradoras asumen la infección por VPH como una enfermedad preexistente y no cubren su tratamiento). En relación al médico debemos considerar los equipos que posee, y la experiencia que tiene en la administración del tratamiento.

La flexibilidad también es una condición necesaria en el tratamiento de una paciente quien tiene VGE. Si la terapia inicial es inefectiva o si la paciente experimenta efectos colaterales significativos, la modalidad de tratamiento deberá ser modificada.

\section{TERAPIAS APLICADAS POR EL MÉDICO}

Podofilina (10-25\%). Es un agente antimitótico que destruye las verrugas induciendo su necrosis. Es uno de los tratamientos contra VGE más antiguos que se conocen. Esta puede ser aplicada a verrugas únicas, pero no reduce el riesgo de absorción sistémica lo cual puede conllevar a una serie de efectos adversos que incluyen supresión de medula ósea, disfunción hepatocelular, compromiso neurológico, alucinaciones, dolor abdominal agudo, por estas razones algunos autores han recomendado el abandono de su práctica (27).
Ácido tricloroacetico (80-90\%). Es recomendado para el tratamiento de las verrugas vaginales y anales. Este agente destruye la verruga por coagulación de sus proteínas a través de una inducción química. El tratamiento no es específico por lo cual puede producir daño a todo tejido que este en contacto. Deberá ser usada una técnica cuidadosa de aplicación debido a que esta solución es de viscosidad más baja comparada con el agua y por lo tanto se esparce rápidamente. Una solución a este problema es crear una barrera con gel de petrolato o ungüento de lidocaína con el fin de evitar que el líquido se esparza a zonas de tejido sano. Una técnica es utilizar un aplicador con punta de algodón, mojarla con la solución y aplicar el agente a la verruga tratando de evitar el tejido sano circundante. Debe asegurarse que toda la solución se haya secado completamente antes de que la apaciente se vista. Debido a que la sensación de ardor es más intensa durante el secado, se puede utilizar un secador de cabello para acelerar este proceso, siempre y cuando la paciente este de acuerdo con este enfoque. Por último, si algo de la solución se derrama en otros tejidos, esta puede ser neutralizada con jabón o bicarbonato de sodio. El exceso de material aplicado a la verruga puede ser absorbido con talco, bicarbonato de sodio o jabón líquido (28).

Terapia quirúrgica. Las verrugas perianales cuyo pedúnculo es delgado pueden ser extirpadas a través de una incisión tangencial bien sea con tijera o bisturí, separando la base de la verruga de la capa superior de la dermis de la piel. Esta es una técnica segura y efectiva (29). La hemostasia es fácilmente lograda con presión, nitrato de plata o solución de Monsel (subsulfato férrico).

Alternativamente se puede utilizar láser de $\mathrm{CO} 2$ o electrocauterio (asa de LEEP) para disecar la verruga. Tales tratamientos obviamente requieren de equipo especial y entrenamiento especializado lo cual incrementa su costo. Después que el anestésico local es aplicado, la verruga puede ser destruida con electrocauterio, pero se debe prestar atención para limitar la profundidad de la destrucción para evitar cicatrices y futuros problemas como vulvodinia o síndromes de hiperestesia (29).

La ablación quirúrgica con láser de $\mathrm{CO} 2$ es también altamente efectiva pero generalmente se reserva para lesiones extensas y aquellas que han demostrado ser resistentes a otras terapias. En tales casos, el procedimiento es realizado en un quirófano bajo anestesia regional. Es importante realizar este procedimiento utilizando un aspirador de humo adecuado debido a la potencial liberación de partículas de VPH (30).

La crioterapia es una técnica que destruye la verruga por congelamiento del agua dentro de la mitocondria. La crioterapia con nitrógeno líquido es recomendada para las verrugas vaginales, verrugas localizadas en el meato uretral y verrugas anales. 
Con esta terapia de citolisis inducida, la licuefacción y destrucción de la verruga puede tomar días o semanas. La aplicación cuidadosa del nitrógeno líquido o una apropiada selección de criosondas es importante para evitar la destrucción del tejido sano que rodea a la verruga. Es común la aparición de dolor después de la aplicación y puede ser reducido con la aplicación de un anestésico tópico local antes de congelar la verruga. La anestesia inyectada puede ser útil para lesiones grandes y/o extensas. El tratamiento puede ser repetido cada 1 a 2 semanas, dependiendo de la respuesta individual al tratamiento. El permitir un tiempo adecuado entre cada aplicación de la terapia ayuda a prevenir las cicatrices hipertróficas de la zona tratada. Para lesiones externas el tratar solo una porción de las lesiones a la vez puede reducir la severidad de los efectos colaterales.

\section{TERAPIAS APLICADAS POR LA PACIENTE}

Podofilox. Al igual que la Podofilina, Podofilox (Condylox ${ }^{\mathrm{TM}}$ ) es una droga antimitótica que destruye a las verrugas induciendo su necrosis. Está disponible como solución que se aplica con los dedos o con un Q-tips. Las aplicaciones se realizan dos veces al día por tres días consecutivos seguido por cuatro días sin tratamiento por un total de cuatro ciclos. Aplican las mismas restricciones a la terapia con Podofilina tal como no usarla en un volumen que exceda los 0,5 ml/por día y limitando el área a tratar a $10 \mathrm{~cm} 2$. El área deberá ser lavada seis a ocho horas después del tratamiento. Puede desarrollarse dolor local moderado o ulceración local. Su seguridad durante el embarazo no ha sido comprobada y por lo tanto se aconseja el uso de un método anticonceptivo efectivo.

Imiquimod (5\%). Es un inmunomodulador que estimula la producción local de citoquinas que destruyen las células infectadas por el VPH. La droga se aplica a las verrugas una vez al día antes de acostarse en días alternos para un total de tres dosis en un periodo de siete días. El régimen puede ser repetido hasta que las lesiones desaparezcan pero por no más de 16 semanas. El área tratada deberá ser lavada con agua y jabón después de 6 a 10 horas después de la aplicación del medicamento. Debido a que Imiquimod está mezclado en un vehículo basado en petrolato, se deberán evitar los condones de látex y los diafragmas vaginales durante su uso. Las parejas deberán esperar hasta que el ungüento sea lavado o deberán usar condones fabricados con polysopreno o poliuretano (31).

\section{NUEVOS TRATAMIENTOS}

Imiquimod (3,75\%). En el año 2010, la Food and Drug Administration (FDA) aprobó el uso de Imiquimod al $3,75 \%$ en crema para el tratamiento de las VGE (32). Imiquimod al 3,75\% está indicado para el tratamiento de las VGE y perianales en pacientes mayores de 12 años. Estudios de Fase II, doble ciego y estudios controlados con placebo han demostrado que Imiquimod al 3,75\% es significativamente más efectivo que el placebo. Adicional a esto, los porcentajes de recurrencia fueron relativamente bajos con un porcentaje de curación de los pacientes de más del $85 \%$, en un protocolo de 12 semanas de seguimiento (33). Imiquimod al 3,75\% deberá ser aplicado a las verrugas durante 8 horas y su frecuencia de administración es una vez al día por dos semanas y se repetirá este ciclo después de dos semanas de descanso. Los porcentajes de curación del Imiquimod al $3,75 \%$ no son tan altos como los de Imiquimod al 5\%. Sin embargo, este nuevo producto tiene algunas ventajas sobre la presentación al $5 \%$ con respecto al cumplimiento del tratamiento por parte de la paciente. El tratamiento con Imiquimod al $3,75 \%$ es significativamente menos prolongado, con una aplicación diaria para un máximo de 8 semanas de tratamiento (34) versus 16 semanas de tratamiento en días alternos para el Imiquimod al 5\% (35). Adicionalmente, la crema al $3,75 \%$ tiene menos efectos colaterales que el Imiquimod al 5\%. Las principales quejas incluyen prurito, sensación de ardor o dolor en la zona de aplicación (36). A diferencia de la crema al 5\%, no se han asociado síntomas sistémicos asociados al uso del Imiquimod al 3,75\%, sin embargo, esta presentación es más costosa que su equivalente al $5 \%$.

Sinecatequinas (Polifenon E). Es un extracto estandarizado de las hojas del té verde (Camellia sinensis). La epigalocatequina galato es la catequina más importante e interviene en múltiples vías de señalización celulares, activando la vía de las caspasas, regulando la expresión de $\mathrm{Bcl}-2$ e inhibiendo la Telomerasa-1. Mediante estos mecanismos se consigue inhibir el ciclo celular, activar la apoptosis, inhibir la transcripción del VPH y activar la inmunidad celular (26). Al combinar todas estas actividades se eliminan tanto las células clínicamente afectadas así como también aquellas con infección subclínica. Las sinecatequinas en crema al 15\% han sido aprobadas en los Estados Unidos para el tratamiento de las verrugas anogenitales externas (Veregen $^{\mathrm{TM}}$ ).

La posología es de 3 aplicaciones diarias durante un máximo de 16 semanas. Estudios aleatorizados y controlados realizados con pacientes de ambos sexos han mostrado tasas generales de regresión de las lesiones entre el 54-65\%, mientras que los pacientes de los grupos placebo obtenían tasas medias del $35 \%(37,38)$.

El efecto del fármaco se empieza a observar clínicamente aproximadamente a partir de la tercera semana de tratamiento, y es más evidente a partir de la cuarta o sexta semana (37). Los signos y/o 
síntomas locales son los efectos indeseados más frecuentes (80\%), sobre todo el eritema y el prurito, y se manifiestan a partir de la segunda o tercera semana de tratamiento $(37,38)$. Pese al alto porcentaje de reacciones, estas son bien toleradas. La reacción inflamatoria, indicativa de la actividad del fármaco, traduce una activación inmunológica local mediada por citocinas proinflamatorias. Se ha observado que los individuos que responden tienen una incidencia superior de reacciones cutáneas locales en comparación con aquellos que no responden al tratamiento (39).

En definitiva, parece un tratamiento efectivo, seguro y bien tolerado, ya que son pocos los pacientes que suspenden el tratamiento debido a los efectos indeseados del preparado. Como contrapartida, es un tratamiento largo que puede prolongarse hasta las 16 semanas y que debe aplicarse 3 veces al día, lo cual puede afectar al cumplimiento del mismo. La mayoría de los estudios aleatorios y controlados excluyen a pacientes con infección por el VIH y trasplantados, hecho que limita los resultados a individuos inmunocompetentes. Tampoco existen datos sobre efectividad en las verrugas internas.

Terapia fotodinámica. La terapia fotodinámica (TFD) con ácido 5-aminolevulinico (AAL) es una técnica relativamente reciente para el tratamiento de las VGE $(40,41)$. Se basa en la respuesta inmunitaria que se produce tras la destrucción tisular inducida por una reacción fototóxica. Se ha observado que el ácido 5-aminolevulínico (ALA) se acumula en mayor cantidad en las células infectadas por el VPH que en la piel normal adyacente (42). Se ha propuesto como una opción para aquellas lesiones rebeldes al tratamiento, o en aquellos casos en los que existen recurrencias pese a haberse realizado un tratamiento correcto. También se ha utilizado en el tratamiento de la Neoplasia Intraepitelial Cervical y vulvar (42).

Se ha demostrado que la TFD sola es más efectiva comparado con el uso de laser de CO2 convencional, siendo la TFD más segura, más efectiva y con porcentajes más bajos de recurrencia (43). Los efectos secundarios son locales y se presentan en forma de dolor, ardor y eritema (35). Parece que el efecto terapéutico de la TFD en los condilomas vendría dado por una rápida activación de las células dendríticas y de los linfocitos CD4 en la piel lesionada (44). La terapia fotodinámica no es una cura para todos los casos, puede ser una terapia adicional a otras terapias destructivas para el tratamiento de la VGE (31).

Vacunas profilácticas que actuarían como tratamiento. Actualmente existen en el mercado dos vacunas profilácticas contra el VPH, una es tetravalente (contra los genotipos 16, 18, 6 y 11) y la otra es bivalente (genotipos 16 y 18). Estas vacunas se basan en la administración de partículas parecidas al virus (VLP's, por sus siglas en inglés) pero sin material genético en su interior, constituidas por la fracción L1 o proteína estructural del virus obtenida a través de recombinación genética. Con esto se logra mimetizar a un verdadero virus, induciendo una respuesta inmune humoral pero sin sus efectos patológicos (45).

Desde que estas vacunas fueron aprobadas para su uso como profilaxis de la infección por VPH en el año 2006 se han reportado casos de remisión de verrugas que habían sido resistentes a diferentes tratamientos $(46,47)$. Hasta la fecha no se conoce el mecanismo exacto por el cual la vacuna profiláctica pudiese provocar esta respuesta o si tal vez se trata de una relación casual por regresión espontanea de las lesiones. Podrían estar implicadas algunas citocinas locales, con inducción de células productoras de interferón o linfocitos CD8 citotóxicos, pero también es posible que los constituyentes no-VPH de la vacuna profiláctica desempeñen algún papel en la desaparición de estas lesiones (47).

Vacunas terapéuticas. Desde hace algunos años se viene explorando la posibilidad de usar tratamientos con vacunas dirigidas contra las oncoproteínas E6/E7 de los VPH oncogénicos (16), pero estarían dirigidas a las lesiones precancerosas y cancerosas provocadas por estos virus oncogénicos, de tal forma que aún faltan estudios que demuestren su efecto sobre las verrugas. Aun no hay suficientes estudios clínicos bien diseñados y con suficientes pacientes que puedan avalar estas observaciones.

\section{CONCLUSIÓN}

La infección por el VPH es una de la ITS con mayor prevalencia aun a pesar de no conocerse con exactitud debido al sub-registro epidemiológico existente. A través del tiempo se han desarrollado una diversidad de tratamientos que van desde el quirúrgico hasta terapias más recientes como los inmunomoduladores y la terapia fotodinámica. Hemos recorrido un largo camino en busca de un tratamiento altamente efectivo para tratar esta patología pero aún queda mucho camino por recorrer y evidentemente la solución está enfocada en tratamientos de tipo inmunológico que logre frenar el desarrollo de estas lesiones y su cura permanente.

\section{REFERENCIAS}

1. Hsueh PR. Human papillomavirus, genital warts, and vaccines. J Microbiol Immunol Infect 2009;42:101-6.

2. Grm HS, Bergant M, Banks L. Human papillomavirus infection, cancer \& therapy. Indian J Med Res 2009;130:277-85.

3. Stanley MA. Genital human papillomavirus infections: Current and prospective therapies. J Gen Virol 2012;93:681-91. 
4. Muñoz N, Kjaer SK, Sigurdsson K, Iversen OE, Hernandez-Avila M, Wheeler CM, Pérez G, Brown DR, Koutsky LA, Tay EH, García PJ, Ault KA, Garland SM, Leodolter S, Olsson SE, Tang GW, Ferris DG, Paavonen J, Steben M, Bosch FX, Dillner J, Huh WK, Joura EA, Kurman RJ, Majewski S, Myers ER, Villa LL, Taddeo FJ, Roberts C, Tadesse A. Impact of human papillomavirus (HPV)-6/11/16/18 Vaccine on all HPV associated genital diseases in young women. J Natl Cancer Inst 2010;102:325-39.

5. Johnston A, Fernando D, MacBride-Stewart G. Sexually transmitted infections in New Zealand in 2003. N Z Med J 2005;118:U1347.

6. Castellsague $X$, Cohet $C$, Puig-Tintore LM, Acebes LO, Salinas J, San Martin M, Breitscheidel L, Remy V. Epidemiology and cost of treatment of genital warts in Spain. Eur J Public Health 2009;19:106-10.

7. Desai S, Wetten S, Woodhall SC, Peters L, Hughes G, Soldan K. Genital warts and cost of care in England. Sex Transm Infect 2011;87:464-8.

8. Patel H, Wagner M, Singhal P, Smita K. Systematic review of the incidence and prevalence of genital warts. BMC Infectious Diseases 2013;13:39.

9. Kliewer EV, Demers AA, Elliott L, Lotocki R, Butler JR, Brisson M. Twenty-year trends in the incidence and prevalence of diagnosed anogenital warts in Canada. Sex Transm Dis 2009;36:380-6.

10. Hoy T, Singhal PK, Willey VJ, Insinga RP. Assessing incidence and economic burden of genital warts with data from a US commercially insured population. Curr Med Res Opin 2009;25:2343-51.

11. Lin C, Lau JT, Ho KM, Lau MC, Tsui HY, Lo KK. Incidence of genital warts among the Hong Kong general adult population. BMC Infect Dis 2010;10:272.

12. Marra F, Ogilvie G, Colley L, Kliewer E, Marra CA. Epidemiology and costs associated with genital warts in Canada. Sex Transm Infect 2009;85:111-5.

13. Wellings K, Collumbien M, Slaymaker E, Singh S, Hodges Z, Patel D, Bajos N. Sexual behaviour in context: a global perspective. Lancet 2006;368:1706-28.

14. Hillemanns P, Breugelmans JG, Gieseking F, Benard $\mathrm{S}$, Lamure $\mathrm{E}$, Littlewood K, Petry KU. Estimation of the incidence of genital warts and the cost of illness in Germany: a cross-sectional study. BMC Infect Dis 2008;8:76.

15. Ávila M, Cavazza ME, Vásquez W, Ortega J, López Y, Correnti M. Genotipificación del virus de papiloma humano en pacientes con condilomas acuminados. Rev Soc Venezolana Microbiol 2008;28:127-33.

16. Wick DA, Web JR. A novel, broad spectrum therapeutic HPV vaccine targeting the E7 proteins of HVP16, 18, 31, 45 and 52 that elicits potent E7specific CD8 T cell immunity and regression of large, established, E7-expressing TC-1 tumors. Vaccine 2011;29:7857-66.

17. Muñoz-Santos C, Pigem R, Alsina M. New treatments for human papillomavirus infection. Actas Dermosifiliogr 2013;104:883-9.
18. CDC. Genital HPV Infection. Hoja de datos. Disponible en: www.cdc.gov/std/HPV/STDFact- HPV.htm \#common. Acceso el 18 de marzo de 2011.

19. Dunne EF, Park IU. HPV and HPV-Associated Diseases. Infect Dis Clin North Am 2013;27:765-78.

20. Hans-Ulrich Bernard. The clinical importance of the nomenclature, evolution and taxonomy of human papillomaviruses. J Clin Virol 2005;(32S):S1-S6.

21. Stanley M. Chapter 17: Genital human papillomavirus infections-current and prospective therapies. J Natl Cancer Inst Monogr 2003;31:117-24.

22. Anderson LA. Prophylactic human papillomavirus vaccines: Past, present and future. Pathology 2012;44:16.

23. Workowski KA, Berman S; Centers for Disease Control and Prevention. Sexually transmitted diseases treatment guidelines, 2010. MMWR Recomm Rep 2010;59(RR-12):1-110.

24. Anic GM, Giuliano AR. Genital HPV infection and related lesions in men. Prev Med 2011;53 Suppl 1:S3641.

25. Daley EM, Perrin KM, McDermott RJ, Vamos CA, Rayko HL, Packing-Ebuen JL, Webb C, McFarlane M. The psychosocial burden of HPV: a mixed-method study of knowledge, attitudes and behaviors among HPV+ women. J Health Psychol 2010;15:279-90.

26. Stockfleth E, Meyer T. The use of sinecatechins (polyphenon E) ointment for treatment of external genital warts. Expert Opin Biol Ther 2012;12:783-93.

27. Von Krogh G, Longstaff E. Podophyllin office therapy against condyloma should be abandoned. Sex Transm Infect 2001;77:409-12.

28. Scheinfeld JD, Lehman DS. An evidence-based review of medical and surgical treatments of genital warts. Dermatol Online J 2006;12(3):5.

29. Kodner CM, Nasraty S. Management of genital warts. Am Fam Physician 2004; 70:2335-42.

30. French L, Nashelsky J, White D. What is the most effective treatment for external genital warts? J Fam Pract 2002;51:312-4.

31. Scheinfeld N. Update on the treatment of genital warts. Dermatol Online J 2013;19(6):18559

32. Berman B, Wolf J. The role of Imiquimod $3.75 \%$ cream in the treatment of external genital warts. Skin Therapy Lett 2012;17:5-7.

33. Baker DA, Ferris DG, Martens MG, Fife KH, Tyring SK, Edwards L, Nelson A, Ault K, Trofatter KF, Liu T, Levy S, Wu J. Imiquimod $3.75 \%$ cream applied daily to treat anogenital warts: combined results from women in two randomized, placebo-controlled studies. Infect Dis Obstet Gynecol 2012;52:828-36.

34. Zyclara ${ }^{\circledR}$ cream, $3.75 \%$ [información del laboratorio fabricante]. Graceway Pharmaceuticals, LLC. Bristol, TN, 2011.

35. Edwards L, Ferenczy A, Eron L. Self-administered topical 5\% Imiquimod cream for external anogenital warts. HPV Study Group. Human Papilloma Virus. Arch Dermatol 1998;134:25-30. 
36. Yanofsky VR, Patel RV, Goldenberg G. Genital warts: a comprehensive review. J Clin Aesthet Dermatol 2012;5:25-36.

37. Tzellos TG, Sardeli C, Lallas A, Papazisis G, Chourdakis M, Kouvelas D. Efficacy, safety and tolerability of green tea catechins in the treatment of external anogenital warts: A systematic review and meta-analysis. J Eur Acad Dermatol Venereol 2011;25:345-53.

38. Tatti S, Swinehart JM, Thielert C, Tawfik H, Mesche$\operatorname{der}$ A, Beutner KR. Sinecatechins a defined green tea extract in the treatment of external anogenital warts: A randomized controlled trial. Obstet Gynecol 2008;111:1371-9.

39. Gross G, Meyer KG, Pres H, Thielert C, Tawfik H, Mescheder A. A randomized, double-blind, four-arm parallel-group, placebo controlled phase II/III study to investigate the clinical efficacy of two galenic formulations of polyphenon $E$ in the treatment of external genital warts. J Eur Acad Dermatol Venereol 2007;21:1404-12.

40. Kreuter A, Wieland U. Lack of efficacy in treating condyloma acuminate and preventing recurrences with the recombinant quadrivalent human papillomavirus vaccine in a case of series of immunocompetents. $J$ Am Acad Dermatol 2013;68:179-80.
41. Liu YX, Zheng HY, Liu XR. 5-aminolevulinic acid-photodynamic therapy for the treatment of cervical condilomata acuminate. Chin Med Sci 2009;24:151-5.

42. Lee Y, Baron ED. Photodynamic therapy: Current evidence and applications in dermatology. Semin Cutan Med Surg 2011;30:199-209.

43. Nucci V, Torchia D, Cappugi P. Treatment of anogenital condylomata with topical photodynamic: report of 14 cases and review. Int J Infect Dis 2010;14 Suppl 3:e280-2.

44. Giomi B, Pagnini F, Cappuccini A, Bianchi B, Tiradritti L, Zuccati G. Immunological activity of photodynamic therapy for genital warts. Br J Dermatol 2011;164:44851.

45. Anderson LA. Prophylactic human papillomavirus vaccines: Past, present and future. Pathology 2012;44:16.

46. Landis MN, Lookingbill DP, Sluzevich JC. Recalcitrant plantar warts treated with recombinant quadrivalent human papillomavirus vaccine. J Am Acad Dermatol 2012;67:e73-4.

47. Kreuter A, Waterboer T, Wieland U. Regression of cutaneous warts in a patient with WILD syndrome following recombinant quadrivalent human papillomavirus vaccination. Arch Dermatol 2010;146:1196-7. 